\title{
Evolución de los embarazos de mujeres infectadas por el virus de la inmunodeficiencia humana
}

\author{
Ricardo Figueroa-D amián, M.C., M. en C..1)
}

\section{Figueroa-Damián R. \\ Evolución de los embarazos de mujeres infectadas por el virus de la inmunodeficiencia humana. Salud Publica Mex 1999;41:362-367.}

\section{Resumen}

Objetivo. Identificar las complicaciones médicas de las embarazadas infectadas por el virus de la inmunodeficiencia humana (VIH) y asintomáticas, y determinar el riesgo que el virus representa para el desarrollo de las mismas. Material y métodos. Se comparó la evolución del embarazo y el resultado perinatal de 44 embarazadas seropositivas al VIH, asintomáticas y que presentaron más de 200 linfocitos CD 4/ $/ \mathrm{mm}^{3}$, con embarazadas control no infectadas; las pacientes se parearon por edad y nivel socioeconómico. Resultados. En 42 (95.4\%) pacientes la vía de contagio fue sexual; 35 (79.5\%) tenían menos de un año de saber que estaban infectadas y $15(34 \%)$ recibieron tratamiento antiviral durante la gestación. Las mujeres infectadas por el VIH presentaron un riesgo mayor de complicaciones infecciosas (RR3.1, IC 95\% 1.9-52), cervicovaginitis (RR2.2, IC 95\% 1- 48) y enfermedades de transmisión sexual (RR18, IC 95\% 2.3-137). El promedio de peso y talla de los recién nacidos y el número de complicaciones neonatales fueron similares entre los grupos comparados. El análisis estratificado mostró que la ausencia de tratamiento antirretroviral y el antecedente de tres o más parejas sexuales incrementaron el riesgo de complicaciones infecciosas. Conclusiones Las embarazadas seropositivas al VIH y asintomáticas no tuvieron un riesgo mayor de complicaciones médicas del embarazo, con excepción de problemas infecciosos.

Palabras clave: embarazo; VIH; cervicitis; enfermedades sexualmente transmisibles; México

\author{
Figueroa-Damián R. \\ Pregnancy outcome \\ in HIV-infected \\ women.
}

Salud Publica Mex 1999;41:362-367.

\begin{abstract}
A bstract
Objective. To identify the medical complications in a cohort of HIV-infected, pregnant women and to determine the risk of having the virus in the development of these complications. Material and methods. A cohort study of 44 HIV-infected and 88 seronegative pregnant women was performed. Pregnancy was followed and perinatal results were compared. HIV-infected women were asymptomatic with CD 4 count $>200 \mathrm{~mm}^{3}$. Patients were matched for age and socioeconomic status. Results. In 42 (95.4\%) of HIV patients the disease had been transmitted sexually; 35 $(79.5 \%)$ had been infected for less than one year and 15 (34\%) received antiviral treatment during pregnancy. HIVinfected patients showed greater risk of infectious disorders (RR3.1, C195\% 1.9-52), cervical infections (RR 2.2, C 195\% 1-48) and sexually transmitted diseases (RR 18,C 195\% 2.3-137). N ewborns showed low birth weight and were premature, and neonatal affections were similar in the two groups compared. Stratified analysis showed that no antiretroviral treatment and more than three sexual partners increase the risk of infections. Conclusions HIV-seropositive, asymptomatic, pregnant women with $>200 / \mathrm{mm}^{3} \mathrm{CD} 4$ count did not show greater medical risk along pregnancy and birth, although higher incidence of infections was detected.
\end{abstract}

Key words: pregnancy; HIV; cervical infection; sexually transmitted diseases; Mexico

(1) Departamento de Infectología e Inmunología, Instituto Nacional de Perinatología. Hospital de Infectología, Servicio de Adultos, Centro Médico $\mathrm{N}$ acional La Raza, Instituto Mexicano del Seguro Social, México.

Fecha de recibido: 30 de abril de 1998 - Fecha de aprobado: 14 de junio de 1999 Solicitud de sobretiros: Dr. Ricardo Figueroa. D epartamento de Infectología, 40. Piso,Torre de Investigaciones, Instituto N acional de Perinatología. Montes U rales 800, colonia Lomas de Virreyes, 11000 México, D.F., México. 
L a pandemia de la infección por el virus de la inmunodeficiencia humana (VIH) y del SIDA afecta a mujeres y niños en el ámbito mundial. En 1996 se estimaba que en el mundo existían más de ocho millones de mujeres infectadas. ${ }^{1}$ En México, hasta el primer trimestre de 1997, se habían notificado, a la Secretaría de Salud, 4012 casos de SIDA en mujeres, de las cuales $69.3 \%$ estaban en edad reproductiva. ${ }^{2}$

En mujeres infectadas por el VIH, el embarazo es de particular importancia, por una parte, por la posibilidad de transmisión vertical de la infección al producto $^{3} \mathrm{y}$, por la otra, por un potencial incremento de la morbilidad perinatal en estas mujeres. Debido al aumento en el número de mujeres en edad reproductiva infectadas por el VIH, la coincidencia de esta infección y el embarazo se ha incrementado. ${ }^{1}$

La mayoría de los estudios sobre embarazadas seropositivas al VIH se han planteado como objetivo identificar la incidencia y los factores de riesgo asociados a la transmisión vertical del VIH, y con menor frecuencia se ha evaluado la morbilidad obstétrica y neonatal de estas mujeres. El objetivo del presente estudio fue identificar la frecuencia y el tipo de complicaciones médicas, obstétricas y neonatales en una cohorte de embarazadas infectadas por el VIH y asintomáticas, además de determinar el riesgo que la infección por el virus representó para la presentación de dichas complicaciones.

\section{Material y métodos}

Se estudió una cohorte de 44 mujeres embarazadas con infección por el VIH, asintomáticas y con una cuenta de linfocitos T CD4 superior a los $200 / \mathrm{mm}^{3}$ desde el punto de vista de la infección viral; estas pacientes, evaluadas prospectivamente, fueron atendidas por el Departamento de Infectología del Instituto Nacional de Perinatología (INPer) en el periodo comprendido de agosto de 1989 a diciembre de 1997. Se formó un grupo control constituido por mujeres embarazadas, sin enfermedades crónicas ni patología obstétrica identificada antes de su ingreso al instituto, y que acudían a consulta de control prenatal. Por cada paciente seropositiva al VIH se incluyeron dos pacientes controles; los grupos se parearon con base en el nivel socioeconómico y la edad.

$\mathrm{Al}$ ingresar a la institución, a las pacientes se les realizó una historia clínica completa, con énfasis en los síntomas prexistentes relacionados con la infección por el VIH.; asimismo, se recopilaron datos demográficos y la historia sexual. Se hizo un seguimiento de la evolución del embarazo, que consistió en consul- tas prenatales cada cuatro semanas, hasta la semana 36 de gestación; posteriormente, se dieron consultas cada dos semanas, hasta el momento del parto.

En cada consulta las pacientes seropositivas al VIH fueron evaluadas en relación con el desarrollo de signos y síntomas de enfermedad o complicaciones obstétricas. Se les realizaron estudios básicos de laboratorio, como biometría hemática, química sanguínea, examen general de orina y citología cervical, además de VDRL (venereal disease research laboratory), antígeno de superficie de hepatitis B (AgsHB), anticuerpos IgM contra citomegalovirus (CMV), cuenta de linfocitos T CD4, cultivo cervicovaginal y ultrasonido obstétrico. Durante el puerperio se realizó una visita hospitalaria diariamente hasta el egreso. A las pacientes con una citología cervical sugestiva de infección por el virus del papiloma humano (VPH), después que finalizó el embarazo y una vez que el cérvix se recuperó del trauma obstétrico, se les realizó colposcopía con toma de biopsia. Se recopiló el número y el tipo de complicaciones que presentaron las embarazadas durante el seguimiento.

A las pacientes controles se les realizaron los mismos estudios y se les dio el mismo seguimiento que a las embarazadas infectadas por el VIH.

A los recién nacidos, hijos tanto de embarazadas seropositivas al VIH como de embarazadas controles, se les realizó un examen clínico que incluyó mediciones antropométricas, identificación de anormalidades morfológicas y signos clínicos de enfermedad. En los casos en que se consideró necesario, se efectuaron los estudios de laboratorio y gabinete pertinentes. Se cuantificó el número y el tipo de complicaciones habidas en los neonatos.

Para fines del estudio, las complicaciones observadas se agruparon en: obstétricas, infecciosas, puerperales y neonatales. Se utilizaron las siguientes definiciones operacionales:

1. Complicaciones obstétricas. Padecimientos propios del embarazo que ponen en riesgo la continuidad del mismo y la integridad del producto.

2. Complicaciones infecciosas. Cualquier tipo de enfermedad infecciosa que se presenta durante el embarazo.

3. Complicaciones puerperales. Problemas directamente relacionados con la resolución del embarazo, que se presentan dentro de los siguientes 10 días después del parto.

4. Complicaciones neonatales. Padecimientos de cualquier tipo ocurridos en los recién nacidos durante los primeros cuatro días de vida. 


\section{A nálisis estadístico}

Se realizó mediante: a) estadística descriptiva; b) pruebas de hipótesis, con $\chi^{2}$ y exacta de Fisher para las variables nominales o discretas, y con $t$ de Student para las variables cuantitativas continuas; se utilizó un nivel de significancia de 0.05 , y c) se cuantificó el riesgo mediante el cálculo de riesgo relativo (RR), con un intervalo de confianza de 95\% (IC95\%), para el cual se obtuvo un valor crudo mediante análisis bivariado y un valor ajustado mediante análisis estratificado. La estratificación se realizó con las siguientes variables: administración de tratamiento antirretroviral, número de parejas sexuales, tiempo de saberse infectada, control prenatal y antecedente de hijos previamente infectados.

\section{Resultados}

El promedio de edad de las embarazadas seropositivas al VIH fue de $25 \pm 5.8$ años, con una mediana de 23 y un intervalo de 17 a 40 años; la mediana de semanas de gestación al ingreso de estas pacientes al INPer fue de 31, con un intervalo de 10 a 39.4 semanas. Las embarazadas controles tuvieron una mediana de edad gestacional al ingreso de 20, con un intervalo de 8 a 26 semanas. Quince $(34 \%)$ mujeres cursaban su primer embarazo; otras $15(34 \%)$, el segundo; ocho (18.2\%), el tercero, y seis $(13.6 \%)$ eran multigestas.

La vía de contagio del VIH fue sexual en 42 (95.4\%) pacientes; en cuatro de ellas se identificó el antecedente de promiscuidad sexual, y en dos, el antecedente de promiscuidad más drogadicción. Del resto de pacientes infectadas por vía sexual, 19 (43.1\%) notificaron dos parejas sexuales estables; 13 (29.5\%), sólo una pareja, y cuatro $(9.1 \%)$, tres parejas. La mediana del número de parejas sexuales en las pacientes del grupo control fue de 2 con un intervalo de 1 a 4 . En relación con el estado de salud de la pareja identificada como infectante, 25 aún estaban vivos y aparentemente sanos, 10 habían fallecido por SIDA, de cinco se desconocía su paradero así como estado de salud y dos estaban vivos pero enfermos. En dos (4.5\%) embarazadas la infección por el VIH se debió a transfusión.

Al momento de ingresar al INPer, 35 pacientes (79.5\%) tenían menos de un año de saber que estaban infectadas; en ocho (18.1\%), el diagnóstico de la infección por el VIH se estableció en los 1-5 años previos al embarazo mediante un estudio, y una paciente tenía más de cinco años de saberse infectada. En 30 mujeres (68.1\%) el diagnóstico de la infección por el VIH se estableció durante el embarazo por medio de unestudio; $14(31.8 \%)$ se embarazaron sabiéndose infectadas, sie- te de las cuales ya tenían un hijo infectado; cabe aclarar que cuatro de estos hijos ya habían fallecido.

Quince embarazadas (34\%) infectadas por el VIH recibieron tratamiento antirretroviral durante la gestación; seis, zidovudina, y nueve, la combinación de zidovudina y didanosina (DDI). De estas pacientes, 13 recibieron además zidovudina durante el trabajo de parto o la cesárea. La mediana del inicio del tratamiento antiviral durante el embarazo fue de 29 semanas de gestación (SDG), con un intervalo de 12 a 36 semanas. Las pacientes que recibieron tratamiento antirretroviral ingresaron al INPer a partir de 1995, año en el que se estableció el tratamiento antirretroviral para todas las embarazadas infectadas por el VIH.

En el cuadro I se muestra la vía de resolución del embarazo en las mujeres seropositivas al VIH y en las pacientes controles. Las indicaciones más comunes de cesárea en las embarazadas VIH (+) fueron amenorrea prolongada y ruptura prematura de membranas (RPM).

Treinta y una $(70.5 \%)$ de las mujeres con infección por el VIH presentaron una o más complicaciones en su embarazo, mientras que 61 pacientes del grupo control $(69.3 \%)$ presentaron algún problema médico durante la gestación (el valor de $p$ no fue significativo). El tipo de complicaciones observadas en cada grupo de pacientes se muestra en el cuadro II. Las mujeres seropositivas al VIH mostraron un riesgo mayor de presentar complicaciones infecciosas durante su embarazo con un RR de 3.1 (IC95\%, 1.9-52); no obstante, sólo una paciente presentó tuberculosis pulmonar, uno de los padecimientos que se incluyen dentro de las infecciones que definen un caso de SIDA. ${ }^{4}$ En el cuadro III se presentan las complicaciones obstétricas en cada uno de los grupos; la frecuencia de aborto fue mayor entre las embarazadas seropositivas al VIH, sin llegar a ser estadísticamente significativa.

De las complicaciones infecciosas (cuadro IV), la cervicovaginitis fue la más frecuente en el grupo de

\section{Cuadro I \\ Vía de RESOlución del embarazo en MUJeRES INFECTADAS POR EL VIH Y SUS CONTROLES. Instituto Nacional de Perinatología. Méxıco, 1989-1997}

\begin{tabular}{|c|c|c|c|c|}
\hline Vía & Embaraz & $\begin{array}{l}\text { sVIH }(+) \\
4\end{array}$ & & \\
\hline & No. & $(\%)$ & No. & $(\%)$ \\
\hline Parto & 28 & (62.9) & 43 & (48.8) \\
\hline Cesárea & 13 & $(29.9)$ & 45 & $(51.2)$ \\
\hline Aborto & 3 & $(6.9)$ & 0 & - \\
\hline
\end{tabular}


embarazadas seropositivas al VIH: en siete casos se trató de candidiasis vaginal, y en cuatro, de vaginosis bacteriana; asimismo, las enfermedades de transmisión sexual (ETS) se presentaron en ocho casos. No se identificaron pacientes con VDRL, AgsHB o IgM contra CMV positivos. La infección por el VIH representó un RR de 2.2 (IC95\% 1-48) para la presencia de cervicovaginitis, y un RR de 18 (IC95\% 2.3-137) para ETS. Dos pacientes con infección por el VIH cursaron con herpes zoster.

\section{Cuadro II \\ INCIDENCIA DE COMPLICACIONES DEL EMBARAZO EN MUJERES SEROPOSITIVAS AL VIH Y SUS CONTROLES. Instituto Nacional de Perinatología. Méxıco, 1989-1997}

\begin{tabular}{|c|c|c|c|c|}
\hline Complicaciones* & $\begin{array}{c}\text { Embarazadas VIH }(+) \\
\frac{n=44}{\text { No. }(\%)}\end{array}$ & $\begin{array}{l}\text { Controles } \\
n=88 \\
\text { No. }(\%)\end{array}$ & $\begin{array}{l}\text { Riesgo relativo } \\
\text { (IC95\%) }\end{array}$ & $p^{\ddagger}$ \\
\hline O bstétricas & $18(40.9)$ & $41(46.6)$ & $0.88(0.6-1.3)$ & NS \\
\hline Infecciosas & $25(56.8)$ & $16(18.2)$ & $3.1 \quad(1.9-52)$ & NS \\
\hline Puerperales & $2 \quad(4.5)$ & $\begin{array}{ll}4 & (4.5)\end{array}$ & $(0.2-5.2)$ & NS \\
\hline N eonatales & $\begin{array}{ll}2 & (4.5)\end{array}$ & $\begin{array}{ll}6 & (6.8)\end{array}$ & $0.7 \quad(0.1-3.2)$ & NS \\
\hline
\end{tabular}

\section{Cuadro III}

Complicaciones obstétricas* en embarazadas INFECTADAS POR EL VIH Y SUS CONTROLES. INSTITUTO Nacional de Perinatología. México, 1989-1997

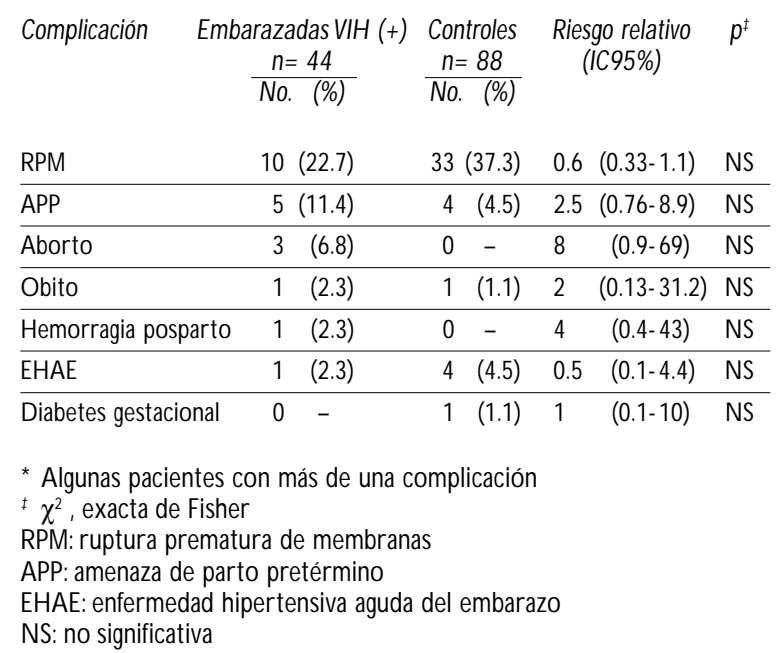

salud pública de méxico / vol.41, no.5, septiembre-octubre de 1999

\begin{abstract}
Cuadro IV
Complicaciones infecciosas* en embarazadas INFECTADAS POR EL VIH Y SUS CONTROLES. INSTITUTO Nacional de Perinatología. México, 1989-1997
\end{abstract}

\begin{tabular}{|c|c|c|c|c|c|}
\hline Complicación & $\begin{array}{l}\text { barazadas VIH (+) } \\
\frac{n=44}{\text { No. }(\%)}\end{array}$ & $\begin{array}{l}\begin{array}{c}\text { Controles } \\
n=88\end{array} \\
\text { No. }(\%)\end{array}$ & & $\begin{array}{l}\text { o relativo } \\
\text { c95\%) }\end{array}$ & $p^{\ddagger}$ \\
\hline Cervicovaginitis ${ }^{\S}$ & $11(25)$ & $10(11.3)$ & 2.2 & $(1-48)$ & 0.05 \\
\hline Infección urinaria & $9(20.5)$ & 8 (9) & 2.2 & $(0.9-5.4)$ & NS \\
\hline ETS & $8(18.2)$ & 0 & 18 & $(2.3-137)$ & 0.001 \\
\hline Herpes zoster & $2 \quad(4.5)$ & 0 & 6 & $(0.6-56)$ & NS \\
\hline Tuberculosis pulmonar & $\begin{array}{ll}1 & (2.3) \\
\end{array}$ & 0 & 4 & $(0.4-42)$ & NS \\
\hline Corioamnioitis & 0 & $3 \quad(3.4)$ & 0.5 & $(0.1-4)$ & NS \\
\hline
\end{tabular}

* Algunas pacientes con más de una infección

$\neq \chi^{2}$, exacta de Fisher

$\S$ Candidiasis, siete casos; vaginosis bacteriana, cuatro casos

ETS: enfermedades de transmisión sexual (infección por el virus del papiloma humano, cuatro casos; herpes genital, dos casos; tricomoniasis, dos casos)

En el grupo de embarazadas VIH (+) hubo cuatro pérdidas fetales, que correspondieron a tres abortos $\mathrm{y}$ un óbito, mientras que en el grupo control sólo hubo una pérdida fetal que consistió en un óbito $(p=0.02)$. Existió una mayor frecuencia de infecciones puerperales entre las pacientes control, aunque no hubo una diferencia estadística entre los dos grupos de pacientes. En relación con la frecuencia de complicaciones neonatales y mortalidad materna no hubo diferencia estadística entre los grupos comparados (cuadro V).

El promedio de peso de los recién nacidos del grupo de seropositivas al VIH fue de $3106 \pm 474 \mathrm{~g}$, mientras que en los neonatos de las madres control el peso promedio fue de $3118 \pm 381 \mathrm{~g}$ (el valor de $p$ no fue significativo); en relación con la talla, el promedio en los hijos de las embarazadas VIH (+) fue de $49.5 \pm 2 \mathrm{~cm}$, contra $49.4 \pm 1.8 \mathrm{~cm}$ de los neonatos del grupo control (el valor de $p$ no fue significativo).

Para establecer la influencia que otras variables tuvieron sobre el desarrollo de las complicaciones infecciosas observadas, se realizó un análisis estratificado, ajustando por diferentes variables potencialmente confusoras. El resultado mostró que el hecho de no haber recibido tratamiento antiviral y el antecedentes de tres o más parejas sexuales incrementaron el riesgo de complicaciones infecciosas; ambas variables fueron modificadoras de efecto. En las embarazadas infectadas por el VIH, el RR de presentar una complicación infecciosa, ajustado por la ausencia de tratamiento antirretroviral durante el embarazo, fue de 12 (IC95\% 5.1-28.6), y este mismo riesgo, ajustado por el ante- 


\section{Cuadro V \\ Complicaciones puerperales, neonatales $Y$ MORTALIDAD EN EMBARAZADAS INFECTADAS POR EL VIH Y SUS CONTROLES. INSTITUTO NaCional de Perinatología. México, 1989-1997}

\begin{tabular}{|c|c|c|c|c|c|c|c|}
\hline $\begin{array}{l}\text { Embara } \\
\qquad \frac{1}{\mathrm{~N}}\end{array}$ & $\begin{array}{l}\text { azad } \\
n= \\
\text { No. }\end{array}$ & $\begin{array}{l}\text { las VIH }(+) \\
\frac{44}{(\%)}\end{array}$ & & $\begin{array}{l}\text { troles } \\
88 \\
(\%)\end{array}$ & & $\begin{array}{l}\text { go relativo } \\
\text { IC95\%) }\end{array}$ & p* \\
\hline Puerperales & & & & & & & \\
\hline Infección H. Qx & 1 & (2.3) & 0 & - & 2 & $(0.13-32)$ & NS \\
\hline Hemorragia & 1 & (2.3) & 0 & - & 2 & $(0.13-32)$ & NS \\
\hline Endometritis & 0 & - & 4 & $(4.5)$ & 0.4 & $(0.1-3.4)$ & NS \\
\hline N eonatales & & & & & & & \\
\hline SFA & 1 & (2.3) & 2 & (2.2) & 1 & $(0.1-10.7)$ & NS \\
\hline $\mathrm{RCIU}$ & 1 & (2.3) & 0 & - & 2 & $(0.13-32)$ & NS \\
\hline Malformación & 0 & - & 1 & $(1.2)$ & 1 & $(0.13-11)$ & NS \\
\hline Fetopatía diabética & 0 & - & 1 & $(1.2)$ & 1 & $(0.13-11)$ & NS \\
\hline Adaptación pulmonar & 0 & - & 1 & $(1.2)$ & 1 & $(0.13-11)$ & NS \\
\hline Policitemia sintomática & 0 & - & 1 & $(1.2)$ & 1 & $(0.13-11)$ & NS \\
\hline D efunciones maternas & 1 & (2.3) & 0 & - & 2 & $(0.13-32)$ & NS \\
\hline $\begin{array}{l}\text { * } \chi^{2} \text {, exacta de Fisher } \\
\text { SFA: sufrimiento fetal ag } \\
\text { RCIU: retardo en crecim } \\
\text { N S: no significativa }\end{array}$ & $\begin{array}{l}\text { gudo } \\
\text { mien }\end{array}$ & to in & & & & & \\
\hline
\end{tabular}

cedente de tres o más parejas sexuales, fue de 5.7 (IC95\% 2.6-12.7).

En el análisis estratificado, el antecedente de menos de tres parejas sexuales mostró ser una variable confusora para el riesgo de adquirir ETS, además de ser un factor protector contra este tipo de complicaciones infecciosas; el RR de presentar ETS entre las embarazadas seropositivas al VIH, ajustado por esta variable, fue de 0.1 (IC95\% 0.02-0.43).

\section{Discusión}

En el ámbito mundial, miles de mujeres están infectadas por el VIH; la mayoría de ellas se encuentran en edad reproductiva, por lo que la coexistencia de infección por el VIH y embarazo no es un evento raro. ${ }^{1} \mathrm{El}$ grupo de pacientes estudiadas en este trabajo no estaban en una fase avanzada de la infección por el VIH; al ingreso, todas eran asintomáticas y tenían cuentas de linfocitos T CD4 superiores a 200 células $/ \mathrm{mm}^{3}$.

En el estudio realizado no se encontró un riesgo mayor de desarrollar complicaciones obstétricas, puerperales o neonatales entre las pacientes seropositi- vas al VIH; otros estudios han descrito que no existe diferencia en la frecuencia de complicaciones del embarazo entre mujeres infectadas por el VIH y asintomáticas, por un lado, y embarazadas controles no infectadas, por el otro. ${ }^{1}$ Gloeb y colaboradores, ${ }^{5}$ al estudiar embarazadas complicadas con infección por el $\mathrm{VIH}$, notificaron una frecuencia de RPM de $15.4 \%$, porcentaje menor al encontrado en nuestro estudio; sin embargo, en relación con el trabajo de parto pretérmino, la frecuencia en ese estudio fue de $34.6 \%$, superior a la identificada en el nuestro. En el informe de Gloeb y colaboradores, ${ }^{5}$ durante el curso del embarazo, $17 \%$ de las mujeres presentaron síntomas relacionados con la infección viral. Minkoff y colaboradores ${ }^{6}$ compararon embarazadas seropositivas al VIH con embarazadas controles seronegativas, sin encontrar diferencia en la frecuencia de complicaciones obstétricas ni neonatales; no obstante, las complicaciones médicas fueron más frecuentes en las seropositivas al $\mathrm{VIH}$. Alger y colaboradores ${ }^{7}$ compararon embarazadas con infección por el VIH pero sin SIDA, con mujeres controles que presentaban similares factores de riesgo pero sin infección por el VIH; los investigadores no encontraron que la infección viral tuviera una influencia adversa sobre el resultado perinatal.

A diferencia de los estudios previamente descritos, el de Temmerman y colaboradores ${ }^{8}$ señala que en un grupo de embarazadas con infección por el VIH, estudiadas en Kenya, existió una mayor frecuencia de partos pretérmino, un menor peso al nacimiento en los productos y un mayor número de complicaciones infecciosas puerperales; la diferencia principal entre este estudio y los anteriormente descritos, es que éste incluyó un número mayor de pacientes en fase avanzada de la infección por el VIH, $43.6 \%$ con sintomas asociados a la infección viral y $4.2 \%$ con SIDA.

Las variables que en nuestro estudio tuvieron diferencia estadística entre los grupos estudiados fueron la frecuencia total de complicaciones infecciosas, el número de casos de cervicovaginitis y el número de ETS; de este modo, se determinó que la infección por el VIH representó un RR para la presentación de estas complicaciones de 3.1 (IC95\% 1.9-52), 2.2 (IC95\% 1-48) y 18 (IC95\% 2.3-137), respectivamente. A pesar del riesgo encontrado para el desarrollo de complicaciones infecciosas en las embarazadas seropositivas al VIH, sólo una de ellas presentó durante su gestación una infección definitoria de caso de SIDA (tuberculosis pulmonar). Nuestros hallazgos coinciden con otros estudios respecto a una mayor frecuencia de complicaciones infecciosas, no asociadas al SIDA, en las embarazadas infectadas por el VIH, como infección urinaria, ${ }^{5}$ ETS $^{5-7}$ o candidiasis vaginal. ${ }^{7}$ 
Minkoff y colaboradores ${ }^{9}$ buscaron infecciones severas en embarazadas con infección por el VIH, y encontraron que las mujeres con una cuenta de CD4 mayor de 300 células $/ \mathrm{mm}^{3}$ no incrementaron su morbilidad infecciosa, mientras que $31 \%$ de las embarazadas con una cuenta de CD4 menor de $300 / \mathrm{mm}^{3}$ presentaron alguna infección severa (infecciones oportunistas, neumonías e infecciones puerperales).

El análisis estratificado que se realizó en el presente estudio mostró que, tanto la ausencia de tratamiento antirretroviral durante el embarazo, como el número de tres o más parejas sexuales, incrementaron el riesgo de complicaciones infecciosas, principalmente debido a un aumento en las infecciones de transmisión sexual. Estos resultados sugieren que el uso de antirretrovirales, además de reducir la transmisión vertical del virus, como ya ha sido demostra$\mathrm{do}^{10}$ también disminuye el riesgo de que las mujeres seropositivas al VIH embarazadas presenten complicaciones infecciosas.

Por lo que se refiere a los neonatos de las pacientes estudiadas, la frecuencia de complicaciones fue similar entre los grupos comparados. Algunos estudios han mostrado que los recién nacidos hijos de madres seropositivas al VIH no tienen un mayor número de complicaciones, ${ }^{6,7}$ pero otros informan de un mayor número de niños con bajo peso al nacimiento y de prematuros. ${ }^{5,8}$ Puede ser que la diferencia entre estos resultados dependa de la población estudiada, aunque quizá también depende del grado de avance de la infección viral en las madres. En un estudio en el que sólo se incluyeron embarazadas con SIDA, ${ }^{11}$ de 27 productos que llegaron al tercer trimestre de la gestación, 14 nacieron prematuros, y de ellos, 11 murieron dentro de las primeras seis semanas de vida.

Existen trabajos en los que habiéndose controlado diversas variables, la infección materna por el VIH se mantuvo como un factor adverso para los productos. Un estudio realizado en Rwanda ${ }^{12}$ mostró que los hijos de madres infectadas por el VIH tuvieron un menor peso al nacimiento, en comparación con recién nacidos de madres sin infección; los autores realizaron un análisis multivariado para controlar variables potencialmente confusoras, y encontraron que el ingreso familiar, la escolaridad de la madre, el estado civil y la edad materna no influyeron en el resultado adverso observado en los recién nacidos. En un estudio multicéntrico realizado en Estados Unidos de América, ${ }^{13}$ mediante un análisis de regresión logística, los autores confirmaron la asociación de prematurez, bajo peso al nacer y muy bajo peso al nacer, con la infección materna por el VIH.
Las embarazadas seropositivas al VIH en etapa aún no avanzada de la infección, asintomáticas y con una cuenta superior a los 200 linfocitos T CD4/ $\mathrm{mm}^{3}$, aparentemente no tienen una morbilidad mayor en $\mathrm{su}$ gestación, pues es parecida a la de embarazadas no infectadas por el VIH y de similar edad y nivel socioeconómico. En este tipo de pacientes las complicaciones infecciosas, si bien se observaron con mayor frecuencia, no consistieron en infecciones de tipo oportunista relacionadas con la infección viral, sino principalmente en infecciones asociadas a la transmisión sexual; en estos casos probablemente la conducta sexual fue la que predispuso tanto a la infección por el VIH como a la presentación de ETS.

\section{Referencias}

1.American College of 0 bstetricians and Gynecologist. Human immunodeficiency virus infections in pregnancy. Int J Gynaecol O bstet 1997;57: 73-80.

2. Consejo Nacional de Prevención y Control del SIDA (CONASIDA). Situación del SIDA en México. Datos actualizados hasta el primer trimestre de 1997. Enferm Infecc Microbiol 1997;17:100-111.

3.Zorrilla C. 0 bstetric factors and mother-to-infant transmission of HIV1. Infect D is C lin N orth Am 1997;11:109-118.

4. World Health O rganization. Acquired immunodeficiency syndrome (AIDS):W HO/CDC case definition for AIDS.W kly Epidemiol Rec 1990; 61:69-76.

5. G loeb J, O 'Sullivan MJ, Efantis J. Human immunodeficiency virus infection in women. The effects of human immunodeficiency virus on pregnancy.Am J O bstet Gynecol 1988;159:756-761.

6. Minkoff $\mathrm{H}, \mathrm{H}$ enderson $\mathrm{C}$, Mendez $\mathrm{H}, \mathrm{G}$ ail M, Holman S,W illoughby A et al. Pregnancy outcome among mothers infected with human immunodeficiency virus and uninfected control subjects. Am J O bstet Gynecol 1990; 163:1598-1604.

7. Alger L, Farley J, Robinson B, H ines S, Berchn J, Johnson J. Interactions of human immunodeficiency virus infection and pregnancy. $O$ bstet $G$ ynecol 1993;82:787-796.

8. Temmerman $M$, Chomba E, N dinya-Achola J, Plummer F, Coppens $M$, Piot P. Maternal human immunodeficiency virus- 1 infection and pregnancy outcome. 0 bstet Gynecol 1994;83:495-501.

9. Minkoff $H, W$ illoughby A, Mendez H, Moroso G, Holman S, Goedert J et al. Serious infectious during pregnancy among women with advanced human immunodeficiency virus infection. Am J O bstet Gynecol 1990;162: 30-34.

10. Connor EM, Sperling RS, G elber R, Kiselen P, Scott G, O 'Sullivan MJ et al. Reduction of maternal-infant transmission of human immunodeficiency virus type-1 with zidovudine treatment. N Engl J Med 1994;331: 1173-1180.

11. Kumar R, Uduman $S$, Khurrana A. Impact of pregnancy on maternal AIDS.J Reprod Med 1997;42:429-434.

12. Bulterys M, C hoo A, Munyemana S, Kurawige JB, N awrocki P, H abimana $P$ et al. Maternal human immunodeficiency virus-1 infection and intrauterine growth:A prospective cohort study in Butare, Rwanda. Pediatr Infect Dis ) 1994;13:94-100.

13. Martin R, Boyer P, Hammill $H$, Peany $H$, Platzker A, Settlage $R$ et al. Incidence of premature birth and neonatal respiratory disease in infants of HIV-positive mothers. J Pediatr 1997;131:851-856. 